Article

\title{
Fatty Acid Profile of Mature Red Blood Cell Membranes and Dietary Intake as a New Approach to Characterize Children with Overweight and Obesity
}

\author{
Iker Jauregibeitia ${ }^{1}\left(\right.$, Kevin Portune ${ }^{1}$, Itxaso Rica ${ }^{2}$, Itziar Tueros ${ }^{1}{ }^{\circledR}$, Olaia Velasco ${ }^{2}$, \\ Gema Grau $^{2}$, Nerea Trebolazabala ${ }^{2}$, Luis Castaño ${ }^{2}$, Anna Vita Larocca ${ }^{3}$, Carla Ferreri ${ }^{4, *(D)}$ \\ and Sara Arranz 1,*(D) \\ 1 AZTI, Food Research, Basque Research and Technology Alliance (BRTA), Parque Tecnológico de Bizkaia, \\ Astondo Bidea, Edificio 609, 48160 Derio, Spain; ijauregibeitia@azti.es (I.J.); kportune@azti.es (K.P.); \\ itueros@azti.es (I.T.) \\ 2 Biocruces Bizkaia Health Research Institute, Cruces University Hospital, CIBERDEM/CIBERER, UPV/EHU, \\ 48903 Barakaldo, Spain; itxaso.ricaechevarria@osakidetza.eus (I.R.); \\ olaia.velascovielba@osakidetza.eus (O.V.); MARIAGEMA.GRAUBOLADO@osakidetza.eus (G.G.); \\ nerea.trebolazabalaquirante@osakidetza.eus (N.T.); luisantonio.castanogonzalez@osakidetza.eus (L.C.) \\ 3 Lipidomic Laboratory, Lipinutragen srl, Via di Corticella 181/4, 40128 Bologna, Italy; \\ annavita.larocca@lipinutragen.it \\ 4 Consiglio Nazionale delle Ricerche, ISOF, Via Piero Gobetti 101, 40129 Bologna, Italy \\ * Correspondence: carla.ferreri@isof.cnr.it (C.F.); sarranz@azti.es (S.A.); Tel.: +(39)-051-639-8289 (C.F.); \\ +(34)-671-750-399 (S.A.); Fax: +(39)-051-639-8350 (C.F.); +(34)-946-572-555 (S.A.)
}

Received: 30 September 2020; Accepted: 8 November 2020; Published: 10 November 2020

\begin{abstract}
Obesity is a chronic metabolic disease of high complexity and of multifactorial origin. Understanding the effects of nutrition on childhood obesity metabolism remains a challenge. The aim of this study was to determine the fatty acid (FA) profile of red blood cell (RBC) membranes as a comprehensive biomarker of children's obesity metabolism, together with the evaluation of their dietary intake. An observational study was carried out on 209 children (107 healthy controls, 41 who were overweight and 61 with obesity) between 6 and 16 years of age. Mature RBC membrane phospholipids were analyzed for FA composition by gas chromatography-mass spectrometry (GC-MS). Dietary habits were evaluated using validated food frequency questionnaires (FFQ) and the Mediterranean Diet Quality Index for children (KIDMED) test. Compared to children with normal weight, children with obesity showed an inflammatory profile in mature RBC FAs, evidenced by higher levels of $\omega-6$ polyunsaturated FAs (mainly arachidonic acid, $p<0.001$ ). Children who were overweight or obese presented lower levels of monounsaturated FA (MUFA) compared to children with normal weight $(p=0.001$ and $p=0.03$, respectively), resulting in an increased saturated fatty acid (SFA)/MUFA ratio. A lower intake of nuts was observed for children with obesity. A comprehensive membrane lipidomic profile approach in children with obesity will contribute to a better understanding of the metabolic differences present in these individuals.
\end{abstract}

Keywords: childhood obesity; inflammation; membrane lipidome; omega-6 fatty acids; red blood cell

\section{Introduction}

Interventions to control obesity have typically consisted of combined strategies including diet, exercise, and behavior therapy [1]. Despite efforts by governments, the food industry, and the science community, obesity and overweight rates keep increasing in both child and adult populations 
worldwide [2,3], demonstrating a need for personalized strategies that guarantee the success of interventions in treating obesity.

The focus on early clinical markers for overweight/obesity onset is, nowadays, a clear research target [4]. A new trend to focus on fats, and specifically the quality of dietary lipids, is crucial for the prevention and treatment of obesity [5]. In this sense, a strong contribution from the molecular approach developed in the last two decades, characterizing fat accumulation, highlights different kinds of signaling occurring in this disease and leading to comorbidities [6]. Since fat accumulation is strictly connected with the quality and quantity of fatty acids (FAs) in human tissues, the lipidomic approach was found to have a key role in describing the scenario of molecular signaling, providing crucial information on the various phases of weight increase, from overweight to obesity [7].

Indeed, membrane fatty acid-based lipidomics has reached a high technology readiness level, developing simple (i.e., inexpensive and high-throughput) and robust analytics of high resolving power, as demonstrated by several applications to diseases [8,9].

Molecular information on membrane lipid composition is of great importance at least for two reasons: (i) the set of membrane fatty acid controls the fluidity and permeability properties as well as the thickness of the bilayer, which are all implicated in the receptor and channel responses [10], and (ii) lipid signaling departs from the fatty acid residues of the membrane phospholipids. Therefore, the fatty acid composition directly describes the cellular predisposition to respond to the various stimuli that arrive from the extracellular environment [11]. Therefore, the balance in the fatty acid composition of the cell membrane leads to the balance in the functions of each individual cell and, hence, of the tissues and the whole organism [12].

It is worth recalling that to measure the lipid composition of blood, different blood compartments have been targeted [13]. Plasma or serum FA levels have been widely analyzed because they reflect short-term dietary fat intake. However, analysis of lipid compositions from mature red blood cell (RBC) membranes offers an advantage over analysis of plasma because these cells last on average 120 days in the blood compared to 3 weeks for platelet or plasma lipids, reflecting better long-term dietary FA intake and tissue conditions [14]. Apart from this, RBCs maintain a more stable FA composition compared to plasma FA levels [15].

It is important to note that although future nutritional intervention studies are necessary to better understand the impact of personalized diet on lipid metabolism in children, lipidomics can help monitor the $\omega-6$ fatty acid content involved in the inflammation pathways that can be accompanied by essential FA deficiencies in the diet, which can be connected to many diseases and tissue malfunctions. As a matter of fact, monitoring the RBC membrane FA profile at the individual level can be an excellent candidate biomarker as it can offer the possibility to follow up the optimal intake, membrane incorporation, and biochemical transformations in order to personalize dietary intervention designed to recover FA deficiencies to prevent or control disease. Fatty acids in phospholipids represent the combination between nutritional and metabolic factors, with a strong contribution of the individual metabolism and condition of the patients.

This study aims to generate knowledge on the importance of different fatty acid families (saturated fatty acids (SFA), monounsaturated fatty acids (MUFA), and polyunsaturated fatty acids (PUFA)) for the cell membrane lipidome in a pediatric population. In this exploratory study, analysis of the RBC membrane shows its potential to provide indications of dietary and metabolic distinctions between children with overweight and obesity that can contribute, in the future, to designing more precise nutritional strategies that may be more effective at correcting the molecular imbalance in obesity.

\section{Materials and Methods}

\subsection{Study Design}

An observational case-control and retrospective study was conducted on 209 children (113 boys and 96 girls) between 6 and 16 years old, recruited from the pediatric endocrinology unit at the Hospital 
Universitario Cruces (Barakaldo, Spain). Children were classified according to body mass index (BMI), using an age and sex-specific pediatric z-score from Orbegozo tables [16]. BMI was taken as a reference to define the different categories, defining normal weight when the standard deviation (SD) of BMI was $-1<\mathrm{SD} \leq+1$, overweight when the $+1<\mathrm{SD} \leq+2$, and obesity when $\mathrm{SD}>+2$. Groups were homogeneously distributed by gender and age. Finally, 107 children with normal weight, 41 children with overweight, and 61 children with obesity were enrolled in the study.

Subjects were excluded if they presented any kind of acute or chronic diseases, were taking medications, or had any presence of metabolic syndrome symptoms or obesity associated to any type of pathology. A physical examination was performed by a pediatrician.

The study protocol was approved by the Euskadi Clinical Research Ethics Committee (permission number PI2016181) and accomplished according to the Helsinki Declaration in 1975, revised in 2013. Subjects under study were included after acceptance (of the parents) to participate in the study and signing of informed consent. All the informed consent documents were signed by their parents, and in the case of children between 12 and 16 years of age, the informed consent was also signed by themselves, according to the Euskadi Ethical Committee and sample biobank laws (Organic Law 3/2018, of December 5, on Protection of Personal Data and guarantee of digital rights; Law 14/2007 on Biomedical Research and RD 1716/2011 of Biobanks).

\subsection{Anthropometric Measures}

Body weight $(\mathrm{kg})$ and height $(\mathrm{cm})$ were measured by standardized methods [17]. Body mass index (BMI) was calculated as weight $(\mathrm{kg})$ divided by the square of the height $\left(\mathrm{m}^{2}\right)$. Anthropometric parameters as well as blood sampling were all conducted by pediatricians during the first visit to the Hospital Universitario Cruces.

\subsection{Food Habits and Nutrient Intakes}

During the first visit, a pediatrician interviewed the participants and collected personal data, including family medical history and information on the history of medication usage. Estimations of food consumption, including dietary diversity and variety, were measured using a quantitative food frequency questionnaire (FFQ), completed online by the parents of each volunteer, except in those cases of adolescents, who were encouraged to complete it themselves. For our study, an adapted FFQ was used, which was previously validated with portion sizes and food groups for the Spanish juvenile population [18-20]. Information about different food items collected from these questionnaires was then analyzed using DIAL ${ }^{\circledR}$ software to translate their intake into their corresponding energy and nutrient composition (UCM \& Alce Ingeniería S.A, Madrid, Spain) (v3.4.0.10).

Dietary habits were also measured using the KIDMED test (Mediterranean Diet Quality Index), a validated questionnaire for the Spanish juvenile population that measures adherence to the Mediterranean diet, which is widely considered to be an optimally healthy diet for most populations [21,22]. According to the KIDMED index, a score of 0-3 reflects poor adherence to the Mediterranean diet, a score of 4-7 describes average adherence, and a score of 8-12 good adherence.

\subsection{Red Blood Cell (RBC) Membrane Fatty Acid Analysis}

The fatty acid composition of mature RBC membrane phospholipids was obtained from blood samples (approximately $2 \mathrm{~mL}$ ) collected in vacutainer tubes containing ethylenediaminetetraacetic acid (EDTA). Samples were shipped to the Lipidomic Laboratory at a controlled temperature and, upon arrival, underwent quality control for the absence of hemolysis. During the blood work-up, before lipid extraction and lipid transesterification to fatty acid methyl esters (FAMEs), the automated protocol includes the selection of mature RBCs, as reported previously [9,23-25]. Briefly, the whole blood in EDTA was centrifuged ( 4000 revolutions per minute (rpm) for $5 \mathrm{~min}$ at $4{ }^{\circ} \mathrm{C}$ ) and the mature cell fraction was isolated based on the higher density of the aged cells [26] and controlled by the use of a cell counter (Scepter 2.0 with Scepter ${ }^{\mathrm{TM}}$ Software Pro, EMD Millipore, Darmstadt, Germany). 
All the subsequent steps were automated and included cell lysis, isolation of the membrane pellets, phospholipid extraction from pellets using the Bligh and Dyer method [27], transesterification to FAMEs by treatment with a potassium hydroxide $(\mathrm{KOH}) /$ methyl alcohol $(\mathrm{MeOH})$ solution $(0.5 \mathrm{~mol} / \mathrm{L})$ for $10 \mathrm{~min}$ at room temperature, and extraction using hexane $(2 \mathrm{~mL})$. The FAMEs were analyzed using capillary column gas chromatography (GC). GC analysis was run on the Agilent 6850 Network GC System (Agilent, USA), equipped with a fused silica capillary column Agilent DB23 $(60 \mathrm{~m} \times 0.25 \mathrm{~mm} \times 0.25 \mu \mathrm{m})$ and a flame ionization detector. Optimal separation of all fatty acids and their geometrical and positional isomers was achieved. Identification of each fatty acid was made by comparison to commercially available standards and to a library of trans isomers of MUFAs and PUFAs. The amount of each FA was calculated as a percentage of the total FA content (relative \%), as described in Section 2.5, being more than $97 \%$ of the GC peaks recognized with appropriate standards.

\subsection{Red Blood Cell Membrane Fatty Acid Cluster}

Twelve FAs were chosen as representative cluster of the main building blocks of the RBC membrane glycerophospholipids and of the three FA families (SFA, MUFA, and PUFA): for SFAs, palmitic acid (C16:0) and stearic acid (C18:0); for MUFAs, palmitoleic acid (C16:1; c9), and oleic acid (C18:1; c9), cis-vaccenic acid (C18:1; c11); for $\omega$-3 PUFAs, eicosapentaenoic acid (EPA) (C20:5) and docosahexaenoic acid (DHA) (C22:6); for $\omega-6$ PUFAs, linoleic acid (LA) (C18:2), dihomo-gamma-linolenic acid (DGLA) (C20:3), and arachidonic acid (AA) (C20:4); for geometrical trans fatty acids (TFAs): elaidic acid (C18:1 t9) and mono-trans arachidonic acid isomers (monotrans-C20:4; $\omega-6$ recognized by standard references as previously described by Ferreri et al. [28]). Considering these fatty acids, different indexes previously reported in the literature [25] were calculated: Omega-3 Index: (\%EPA + \%DHA) an index suggested as a cardiovascular disease risk factor; (\%SFA/\%MUFA) index related with membrane rigidity; inflammatory risk index (\% $\omega-6) /(\% \omega-3)$; PUFA balance $(\% E P A+\% D H A) /$ total PUFA $\times 100$; free radical stress index (sum of trans-18:1 + summary $(\Sigma)$ of monotrans 20:4 isomers); unsaturation index (UI) $(\%$ MUFA $)+(\% \mathrm{LA} / 2)+(\% \mathrm{DGLA} / 3)+(\% \mathrm{AA} / 4)+(\% \mathrm{EPA} / 5)+(\% \mathrm{DHA} / 6) ;$ peroxidation index (PI) $(\%$ MUFA $/ 0.025)+(\%$ LA $)+(\%$ DGLA $/ 2)+(\% \mathrm{AA} / 4)+(\% \mathrm{EPA} / 6)+(\% \mathrm{DHA} / 8)$.

Additionally, the enzymatic indexes of elongase and desaturase enzymes, the two classes of enzymes of the MUFA and PUFA biosynthetic pathways, were inferred by calculating the product/precursor ratio of the involved FAs.

\subsection{Statistical Analysis}

A power calculation was performed using $\mathrm{G}^{*}$ power software (v3.1.9.7., Heinrich-Heine-University, Düsseldorf, Germany), to determine the total sample size for analysis of covariance (ANCOVA) fixed effects, main effects, and interactions. A priori, we expected a medium effect size $\mathrm{f}=0.25$, (as the ratio of the variation among the group means to the average variation among subjects within each group as measured by their standard deviations), using an alpha $=0.05$ as probability of the type I error, to have a $95 \%$ confidence for significative results, and beta $=0.2$, as the acceptable probability of type II errors, concluding in a 0.8 power (where power is equal to 1-beta). It is estimated that a lower value would imply too great a risk of incurring a type II error. A higher value would imply excessively expanding the sample [29]. The total sample size required was 158, which corresponds with 53 participants for each group.

Differences between groups for nutrient intake, food group intake, and KIDMED test were determined by conducting a Kruskal-Wallis test because the data were not normally distributed. Normal data distribution was assessed by Shapiro-Wilk's test or/and the Kolmogorov-Smirnov test. Subsequently, Dunn's (1964) test was performed for post hoc comparisons. A Bonferroni correction for multiple comparisons was made, to correct for the increased risk of type I error.

An analysis of covariance test (ANCOVA) was run to determine the differences between RBC membrane fatty acids from children with normal weight, overweight, and obesity, after controlling for variables selected as potential confounders, such as age, gender, and dietary macro- and 
micronutrient intake. Posthoc analysis was performed with a Bonferroni adjustment for multiple comparisons. First, a principal component analysis (PCA) was run on 18 dietary nutrient intake variables (individual FAs, families (SFA, MUFA, and PUFA), total lipids (\%Energy), carbohydrates, fiber, proteins, and calories), obtained with DIAL software (v3.4.0.10, Department of Nutrition (UCM) \& Alce Ingeniería, S.L., Madrid, Spain) after transforming the information about food items from FFQ questionnaires into micro- and macronutrient values, in order to reduce and simplify the dimensions of these variables and use the generated factors as diet covariates [25]. Kaiser-Meyer-Olkin (KMO) and Bartlett's test of sphericity were used to verify the sampling adequacy for the analysis. The PCA revealed three components that had eigenvalues greater than one and which explained $83.74 \%$ of the total variance. These components were included in the ANCOVA analysis as diet covariates. The level of significance was set at $p<0.05$.

In order to establish correlations between the RBC membrane FA profile and the dietary intake and other parameters measured in this study, due to the non-normality of the data of most of the variables, Spearman's rank-order correlation was conducted. In those cases where all variables had a normal distribution, a Pearson product-moment correlation was run. All statistical analyses were performed using SPSS (IBM Corp. v24.0, Armonk, NY, USA).

\section{Results}

\subsection{Dietary Intake}

Table 1 shows dietary intake according to food categories calculated via food questionnaires. The diet of children with obesity was characterized by lower intake of cereals $(p=0.04)$, dairy products $(p=0.05)$, and nuts $(p=0.01)$, compared to children with normal weight. The overweight group only showed significant differences of lower intake of cereals compared with the group of children with normal weight $(p=0.004)$. Regarding diet quality, the KIDMED questionnaire was conducted to measure the adherence of different groups to the Mediterranean diet. As we can see in Table 1, only children with normal weight achieved good adherence (KIDMED score $\geq 8$ ), while children with overweight and obesity showed mild adherence to the Mediterranean diet (less than 8 points and equal to or greater than 4 points) [21]. Even so, children with normal weight just had a difference of one point in the KIDMED scale compared with the other groups and no statistically significant differences were observed, $(p=0.07)$. KIDMED and BMI showed a statistically significant slight negative correlation (Pearson's Correlation coefficient $\left.\mathrm{r}_{\mathrm{S}}(98)=-0.198, p=0.004\right)$. The KIDMED index did show some slight correlation with RBC membrane AA, EPA, and DHA levels $\left(\mathrm{r}_{\mathrm{s}}(98)=-0.183, p=0.010, \mathrm{r}_{\mathrm{s}}(98)=0.195\right.$, $p=0.006$, and $\mathrm{r}_{\mathrm{S}}(98)=0.227, p=0.001$, respectively).

Table 2 shows the differences among three groups in macronutrients and individual fatty acid daily intake expressed as \% of Kcal. No differences among groups were observed for macronutrients, except for the group of children with normal weight that showed a lower intake of total lipids compared with the overweight group $(p=0.01)$. Considering the intake of specific fatty acids, the children with normal weight reported a higher intake of C16:0 and total SFAs compared to the groups with overweight and obesity, but no other differences were observed. 
Table 1. Food group intakes.

\begin{tabular}{|c|c|c|c|c|c|c|c|}
\hline \multirow[t]{2}{*}{ Food Groups (g/day) } & \multirow{2}{*}{$\begin{array}{c}\text { Children with Normal } \\
\text { Weight (NO) } \\
n=107 \\
\text { Med (Q1-Q3) }\end{array}$} & \multirow{2}{*}{$\begin{array}{c}\text { Children Who Are } \\
\text { Overweight (OV) } \\
n=41 \\
\text { Med (Q1-Q3) }\end{array}$} & \multirow{2}{*}{$\begin{array}{c}\text { Children with } \\
\text { Obesity (OB) } \\
n=61 \\
\text { Med (Q1-Q3) }\end{array}$} & \multirow{2}{*}{$\begin{array}{l}\text { Kruskal-Wallis } \\
\text { H Test }(p)\end{array}$} & \multicolumn{3}{|c|}{$\begin{array}{l}\text { Post hoc Pairwise } \\
\text { Comparison }\left(p^{*}\right)\end{array}$} \\
\hline & & & & & NO:OV & NO:OB & OV:OB \\
\hline Fruits & $423(297-532)$ & $354(273-509)$ & $419(272-603)$ & 0.50 & & & \\
\hline Vegetables & $159(96-259)$ & $154(77-250)$ & $141(74-232)$ & 0.49 & & & \\
\hline Cereals & $161(118-210)$ & $127(96-171)$ & 139 (105-188) & 0.01 & 0.01 & 0.11 & 0.95 \\
\hline Legumes & $91(54-102)$ & $80(51-102)$ & $75(48-96)$ & 0.52 & & & \\
\hline Olive oil & $15(15-37)$ & $15(12-15)$ & $15(12-37)$ & 0.26 & & & \\
\hline Dairy products & $325(255-512)$ & $314(226-329)$ & $302(207-358)$ & 0.10 & & & \\
\hline Eggs & $15(15-34)$ & $15(15-34)$ & $15(15-34)$ & 0.36 & & & \\
\hline Red meat & $21(21-21)$ & $21(21-50)$ & $21(21-50)$ & 0.31 & & & \\
\hline White meat & $50(21-50)$ & $50(36-50)$ & $50(21-50)$ & 0.15 & & & \\
\hline Dried fruits and nuts & $2.1(0-6.4)$ & $1.1(0-6.4)$ & $1.1(0-2.1)$ & 0.03 & 0.73 & 0.03 & 0.96 \\
\hline Lean fish & $27(27-27)$ & $27(27-27)$ & $27(13-27)$ & 0.613 & & & \\
\hline Oily fish and shellfish & $27(13-27)$ & $27(9-31)$ & $27(11-31)$ & 0.55 & & & \\
\hline Sugary drinks & $18(0-45)$ & $21(0-54)$ & $16(0-54)$ & 0.95 & & & \\
\hline Juices & $80(27-250)$ & $107(27-196)$ & $80(27-250)$ & 0.95 & & & \\
\hline KIDMED score & $8(7-9)$ & $7(6-9)$ & $7(6-9)$ & 0.07 & & & \\
\hline
\end{tabular}


Table 2. Dietary daily intake expressed as \% of energy (\%E).

\begin{tabular}{|c|c|c|c|c|c|c|c|}
\hline \multirow[t]{2}{*}{ Variables } & \multirow{2}{*}{$\begin{array}{c}\text { Children with Normal } \\
\text { Weight (NO) } \\
n=107 \\
\text { Med (Q1-Q3) }\end{array}$} & \multirow{2}{*}{$\begin{array}{c}\text { Children Who Are } \\
\text { Overweight (OV) } \\
n=41 \\
\text { Med (Q1-Q3) }\end{array}$} & \multirow{2}{*}{$\begin{array}{c}\text { Children with } \\
\text { Obesity (OB) } \\
n=61 \\
\text { Med (Q1-Q3) }\end{array}$} & \multirow[t]{2}{*}{$\begin{array}{c}\text { Kruskal-Wallis H } \\
\text { Test }(p)\end{array}$} & \multicolumn{3}{|c|}{$\begin{array}{l}\text { Post Hoc Pairwise } \\
\text { Comparison }\left(p^{*}\right)\end{array}$} \\
\hline & & & & & NO:OV & NO:OB & OV:OB \\
\hline \multicolumn{8}{|c|}{ Macronutrients } \\
\hline Calories (Kcal/day) & 2058 (1749-2376) & $1983(1516-2335)$ & 1916 (1709-2167) & 0.18 & & & \\
\hline Proteins (\%E) & $16.3(15.0-17.7)$ & $16.8(15.3-18.5)$ & $16.4(14.9-17.3)$ & 0.42 & & & \\
\hline Carbohidrates (\%E) & $46.7(43.2-49.9)$ & $48.1(43.9-53.3)$ & $46.7(43.4-51.1)$ & 0.19 & & & \\
\hline Simple sugars (\%E) & $20.9(18.5-23.8)$ & 20.9 (17.9-24.8) & $21.8(18.9-25.0)$ & 0.42 & & & \\
\hline \multicolumn{8}{|c|}{ Individual FA (\% E) } \\
\hline C14:0 & $1.0(0.8-1.2)$ & $0.8(0.6-1.1)$ & $0.9(0.7-1.1)$ & 0.02 & 0.06 & 0.08 & 1.0 \\
\hline $\mathrm{C} 16: 0$ & $6.3(5.7-7.1)$ & $5.8(5.1-6.7)$ & $5.8(5.2-6.7)$ & 0.01 & 0.04 & 0.03 & 1.0 \\
\hline C18:0 & $2.4(2.1-2.7)$ & $2.2(1.9-2.5)$ & $2.2(2.0-2.6)$ & 0.05 & & & \\
\hline Tot. SFA & $9.7(8.7-10.9)$ & $9.0(7.8-10.1)$ & $9.0(7.9-10.4)$ & 0.004 & 0.02 & 0.04 & 1.0 \\
\hline C16:1 & $0.51(0.46-0.58)$ & $0.49(0.42-0.54)$ & $0.50(0.42-0.60)$ & 0.1 & & & \\
\hline C18:1 & $14.2(11.4-16.5)$ & $12.3(10.1-14.8)$ & $13.3(11.0-17.3)$ & 0.08 & & & \\
\hline Tot. MUFA & $14.7(11.9-17.1)$ & $12.7(10.5-15.2)$ & $13.9(11.4-17.8)$ & 0.07 & & & \\
\hline C20:4 & $0.04(0.03-0.05)$ & $0.04(0.03-0.06)$ & $0.04(0.03-0.05)$ & 0.78 & & & \\
\hline Tot. $\omega 6$ & $3.5(3.0-4.4)$ & $3.7(2.8-4.5)$ & $3.4(3.1-5.0)$ & 0.97 & & & \\
\hline$>\mathrm{C} 18: 3$ & $0.52(0.50-0.61)$ & $0.50(0.46-0.54)$ & $0.52(0.45-0.58)$ & 0.44 & & & \\
\hline C20:5 (EPA) & $0.07(0.04-0.1)$ & $0.07(0.02-0.11)$ & $0.07(0.04-0.1)$ & 0.88 & & & \\
\hline C22:5 (DPA) & $0.017(0.011-0.024)$ & $0.017(0.006-0.025)$ & $0.016(0.009-0.025)$ & 0.59 & & & \\
\hline 22:6 (DHA) & $0.14(0.09-0.19)$ & $0.13(0.05-0.20)$ & $0.13(0.09-0.19)$ & 0.84 & & & \\
\hline Tot. $\omega 3$ & $0.8(0.7-1.0)$ & $0.8(0.6-0.9)$ & $0.8(0.6-0.9)$ & 0.36 & & & \\
\hline Tot. PUFA & $4.3(3.8-5.3)$ & $4.5(3.5-5.4)$ & $4.3(3.6-5.5)$ & 0.96 & & & \\
\hline$\omega-6 / \omega-3$ & $4.6(4.0-5.4)$ & $4.8(3.7-6.8)$ & $4.9(4.0-6.7)$ & 0.25 & & & \\
\hline
\end{tabular}

Data are expressed as medians and quartile 1 and quartile 3 (Med Q1-Q3. Not normally distributed variables.

PUFA-polyunsaturated fatty acid, (\%E)-\%Energy. * Pairwise comparison conducted with a Bonferroni adjustment. 


\subsection{RBC Membrane Fatty Acid Profile}

In order to compare RBC FA profiles between groups, a one-way ANCOVA was conducted using age, sex, and dietary intake as covariates to adjust the error made by those confounding factors (Table 3). The group with obesity showed higher levels of stearic acid $(p=0.03)$ and total SFA $(p=0.03)$ than the normal weight group. Oleic acid and total MUFA levels in the groups with obesity and overweight were lower compared with the children with normal weight. Regarding $\omega-6$ FA, linoleic acid was higher for the normal weight group compared to children with obesity $(p=0.03)$, but dihomo- $\gamma$-linolenic acid (DGLA) and arachidonic acid levels were higher for children with obesity compared with the children with normal weight ( $p=0.002$ and $p=0.0003$, respectively). Individual and total $\omega-3$ levels did not show significant differences. The SFA/MUFA ratio was higher for children with obesity and overweight compared with children with normal weight $(p=0.001$ and $p=0.03$, respectively), and for the $\omega-6 / \omega-3$ ratio, children with obesity and overweight showed higher but not statistically significant values compared with the children with normal weight $(p=0.09$ and $p=0.1$, respectively).

With respect to enzymatic activity, $\triangle 9 \mathrm{D}$ was lower for children with obesity, indicating the hypoactivity of this enzyme to convert stearic acid (C18:0) to oleic acid (9c C18:1).

In order to explore possible relationships between dietary intake components and RBC lipid profile, a Spearman's rank-order correlation was carried out. Only dietary EPA, DPA, and DHA showed mild correlations with levels of EPA in the RBC $\left(\mathrm{r}_{\mathrm{S}}(98)=0.33 p<0.0001, \mathrm{r}_{\mathrm{S}}(98)=0.363 p<\right.$ 0.0001 , and $r_{\mathrm{s}}(98)=0.313 p<0.0001$, respectively).

At the same time, dairy products and cereal intake were higher for children with normal weight compared to children with obesity, but neither showed significant correlations with the RBC membrane FAs.

Table 3. Red blood cell (RBC) membrane fatty acid profile.

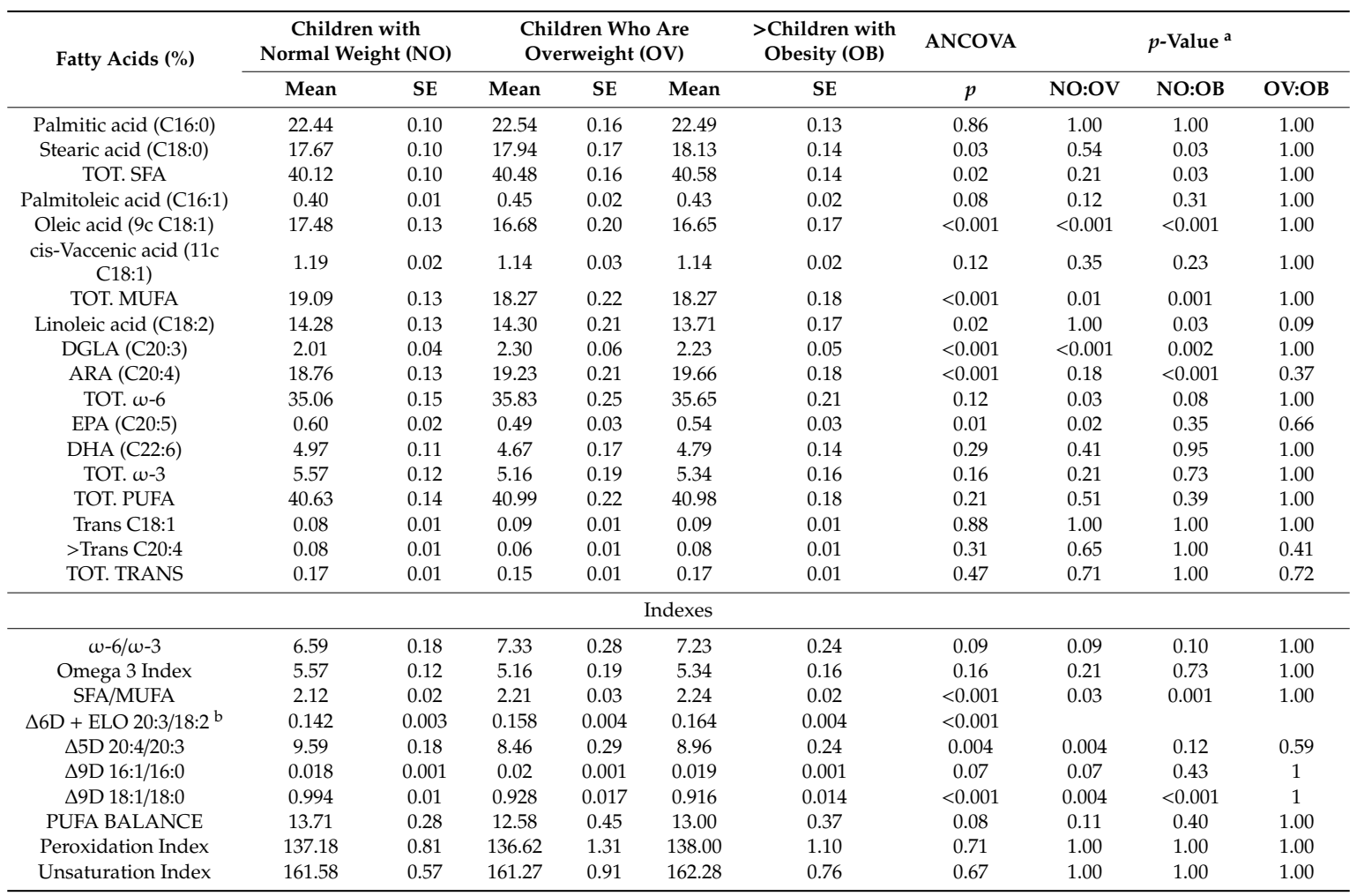

Data are presented as mean \pm standard error (SE). Adjusted for age, sex, and dietary components, extracted from the principal component analysis of dietary nutrient intake (individual FAs, families (SFA, MUFA, and PUFA), total lipids (\%Energy), carbohydrates, fiber, proteins, and calories). ${ }^{a}$ Post hoc tests were conducted with a Bonferroni adjustment. ${ }^{\mathrm{b}}$ Levene's test of homogeneity of variance was not met. 


\section{Discussion}

Lipidomic monitoring of mature RBC membranes evaluated in this study contributes to highlighting the importance of each fatty acid class as a molecular parameter to better understand the lipidomic pathways connected with childhood obesity. Our results indicate molecular inflammation derived from unbalanced levels of membrane fatty acids and dysregulation of desaturase enzymatic activity as key parameters for the metabolic outcome in obesity.

Different studies have been published reporting RBC membrane lipid profiles of adult populations with overweight and obesity [30,31] and a few, also, on child populations with obesity [32].

In a meta-analysis study published by Fekete et al. [33], the FA profiles from different blood fractions were analyzed in order to determine the long-chain PUFA status in obesity. In total plasma lipids and phospholipids, high variability in individual FA levels was observed throughout the different studies analyzed. All the studies agreed that differentiated lipid profiles were observed for subjects with overweight and obesity compared to subjects with normal weight, characterized by a greater alteration in $\omega-6$ FA. In agreement with our results, all the biomarkers analyzed in this meta-analysis showed increased levels of DGLA and decreased LA for the population with overweight and/or obesity. This meta-analysis could not find any significant result for AA, despite increased levels of AA in adipose tissue, which has been previously associated with obesity [34-36]. However, our results revealed significant increased levels of AA in RBC membranes in children with obesity. This heterogeneity points towards a need for a more precise biomarker to characterize and compare different population groups, highlighting the advantages of choosing mature RBC membrane as a representative of nutritional and metabolic contributions [11].

Our results can be seen also in view of other studies that have also analyzed RBC membranes. A study on children with overweight and obesity with metabolic syndrome from 5 to 18 years of age in an Italian population showed similar FA levels of total $\omega-6$ and total SFAs, whereas for all the other measured PUFAs and indexes, differences could be observed [32]. The variability in RBC FA between different countries [37] might be the reason, and this is an important point to emphasize in our approach, which proposes common features of an automatized procedure for cell sampling and membrane isolation and of a precise cluster of fatty acids to analyze.

To our knowledge, this is the first time that a systematic approach was employed that analyzes FAs from isolated mature RBC membranes in pediatric populations with overweight and obesity compared to children with normal weight in order to identify specific characteristics of the fatty acid profile for childhood obesity. Furthermore, as the composition of the RBC membrane is substantially affected by diet and metabolism, the elimination of the effect of diet as a confounding variable in our ANCOVA analysis allows a more robust and realistic examination of the effects of metabolic status of the obesity condition on the RBC FA profile.

According to data obtained in our study, the group with obesity is characterized by an increase in $\omega-6$ fatty acids due to the higher levels of AA and, at the same time, of DGLA, but $\omega-3$ mediated signaling also has to be balanced. Omega-6 FAs have been previously described in the literature as precursors of proinflammatory mediators $[38,39]$ that act through different mechanisms on inflammatory processes. Unlike the other $\omega-6$ FAs, LA showed lower levels for the group with obesity compared with the children with normal weight. Higher RBC LA levels have been linked with improved body composition, insulin resistance, and lower levels of inflammatory markers in previous studies. This disequilibrium of PUFA metabolism towards $\omega-6$ FAs seems to contribute to excessive adipose tissue development and represents, itself, an emerging risk factor for obesity [40,41].

Previous studies have shown an inverse correlation between $\omega-3$ intake and AA levels in RBCs that can be due the competition of the $\Delta 6$-desaturase [42]. Although we did not observe differences in the dietary intake of $\omega-3$, nor in RBC $\omega-3$ levels, but considering that AA levels appear to be enhanced in obesity, an increase in $\omega-3$ consumption in the population with obesity can be a crucial dietary recommendation in order to counteract proinflammatory precursors linked with the disease. 
Regarding SFA and MUFA levels, an altered ratio in the group with obesity can be observed, mainly due to lower levels of oleic acid and higher levels of stearic acid in the group with obesity compared with the children with normal weight. The enzymatic activity of $\Delta$-9-desaturase or stearoyl-CoA desaturase-1 (SCD1), measured indirectly by the ratio between oleic and stearic acids, showed a lower activity for the group with obesity. The overall picture that comes from examination of the fatty acid remodeling occurring in obesity highlights the role of de novo lipogenesis with the formation of saturated fatty acids (SFA) and their enzymatic transformation to monounsaturated fatty acids (MUFA), connected with the functioning of delta-9 desaturase and the corresponding gene expression (SCD1, Stearoyl CoA Desaturase). As a consequence, the main fatty acid biomarkers of weight increase are MUFAs, such palmitoleic acid ( 9 cis-16:1) and oleic acid ( 9 cis-18:1), the former being considered for its role as a lipokine [43] and the latter being considered the main fatty acid accumulating in adipose tissue as triglycerides [44-48].

Although higher dietary intake of SFAs was observed in the normal weight group compared with the group with obesity, the SFA level in RBC membranes was slightly higher in the study population with obesity. Possible explanations for this result, as explained above, could be due to the greater activity of SCD1 in the children with normal weight, which may have converted higher proportions of SFAs to MUFAs, as reflected in higher oleic acid levels in this group. At the same time, the SFA/MUFA ratio, which is correlated with increased membrane rigidity, appears in higher levels in the group with obesity $[12,49]$.

Precision nutrition based on molecular data considers that the assessment of dietary patterns provides a more reliable picture of real food intake compared to the assessment of individual macronutrient intake [50]. Links between dietary patterns and RBC lipid composition have been considered in our study to provide information that could be useful for more precise nutritional recommendations. Dietary patterns of children with normal weight were characterized by higher intake of nuts compared with the group with obesity and a higher intake of cereals compared with the overweight group, but neither food groups showed correlations with the RBC membrane FAs. However, different epidemiological and nutritional clinical trials conducted in adults have reported an inverse relationship between nut consumption and body mass index (BMI) [51-53], associated with several health benefits, such as antioxidant, hypocholesterolemic, cardioprotective, anticancer, anti-inflammatory, and antidiabetic benefits, among other functional properties [54,55]. Previously published results based on self-reported intakes using food frequency questionnaires pointed out that a high intake of grains could be protective against obesity [56,57]. Regarding diet quality, measured by the KIDMED test, statistically significant differences were not observed. Even so, a tendency of a higher adherence to Mediterranean diet in the children with normal weight, compared with the groups with overweight and obesity, can be observed $(p=0.07)$, and these results agree with other studies with pediatric populations with overweight and obesity [58].

In our opinion, there are several points to be considered for the identification of a fat biomarker in obesity, as well as in other physio-pathological states. The first focus is on the choice of the sample to examine: (i) mature red blood cells are representative for both functional and structural roles of the fatty acid residues which compose its membrane phospholipids; (ii) the fatty acids in phospholipids represent the combination between nutritional and metabolic factors, with strong contribution to the individual metabolism and condition of the patients.

The second focus is on the variations in the fatty acid residues of membrane phospholipids, which point to a differentiation between the overweight and obese status: (a) oleic acid is reduced in both subjects with overweight and those with obesity, which is interesting since, in adults, it is known that the decrease in this fatty acid is correlated with weight increase [31]. The role of the FFQ checked in our study clarified that there is no increase in SFA intake in children with obesity, thus shifting the attention to the metabolism of children with obesity and enzymatic functioning. It should be noted that in overweight and obesity, the decrease in desaturase delta-9 activity is significant, thus suggesting a shift in the enzyme functioning toward the SFA pathway, and (b) the role of the omega-6 pathway 
for the arachidonic acid increase is clearly shown in the comparison between children with normal weight and children with obesity, showing that in obesity, the increase in inflammatory signals could be crucial to be controlled in order to treat metabolic deficiencies related to childhood obesity.

This is an exploratory study that highlights some aspects to be further assessed in larger populations but also gives an indication for dietary and metabolic distinctions between children with overweight and obesity, to be further explored with intervention studies. At the same time, the indirect measurement of enzyme activity by the ratio between product and precursors, although very popular, could be considered as a limitation of the study and should be measured directly to emphasize and reaffirm the conclusions obtained. The use of an FFQ is also a limitation of the study as this type of questionnaire usually underestimates dietary intake [59]. Regarding the sample size, the authors are aware that the overweight group did not achieve the expected sample size, which was attributed to difficulties in recruiting, typically observed in human studies and especially in recruiting children; therefore, there might not have been enough power to detect differences in the overweight group and this could have led to a type II error. Future work should be focused on increasing the study sample size to achieve a greater statistical power. In order to compare with other studies that use international tables for determining obesity, such as the International Obesity Task Force (IOTF) or the World Health Organization (WHO), using the Spanish tables described above can be seen as a limitation, even if they better describe our study population. We can summarize that the presented study showed an altered RBC FA profile of the pediatric population with obesity, characterized by two main features: (i) an increased $\omega-6$ molecular contribution, although no differences in PUFA dietary intake between groups were observed, which can create a predisposition for unbalanced signaling that departs from membranes; (ii) higher SFAs in the RBC membranes of children with obesity, contrary to the SFA intake that was found to be higher in controls, which can perturb membrane organization. Both results highlight the crucial role of molecular diagnostics for precise evaluation of patient status. Indeed, the lipidomic analysis of mature RBCs provides a systematic, automatized approach for the characterization of the lipid composition in these cells from the pediatric population with obesity, which can provide molecular insights to assist further development of precise and personalized nutritional strategies. Restoration of the optimal levels of each individual fatty acid, families, and ratios appears to be an important strategy to be considered in the treatment of potentially metabolic deficiencies related to childhood obesity from a nutritional point of view. Future studies, mainly nutritional intervention studies with children, are needed to elucidate an adequate selection among the types of fats that must be ingested, with the crucial target of the recovery of the homeostatic levels of the cell membrane, for proper functioning, both of signaling and metabolic pathways.

Author Contributions: Conceptualization, S.A. and I.J.; methodology, S.A., I.R., L.C. and C.F.; formal analysis, I.J., N.T., G.G., O.V. and A.V.L.; investigation, I.J. and S.A.; data curation, I.J. and K.P.; writing-original draft preparation, I.J. and S.A.; writing - review and editing, I.J., S.A., I.T. and C.F.; supervision, S.A. and C.F. All authors have read and agreed to the published version of the manuscript.

Funding: This work was supported by the Department of Environment: Territorial Planning: Agriculture and Fisheries of the Basque Country Government (ELKARTEK 2017: and Innovation Fund 2017); the Department of Health of the Basque Government (2017222033: OBESIA 2016-2019); the Centre for the Development of Industrial Technology (CDTI) of the Spanish Ministry of Science and Innovation under the grant agreement: TECNOMIFOOD project (CER-20191010); the INC (INTERNATIONAL NUT AND DRIED FRUIT COUNCIL) under the grant agreement OBINUT project (2016(II)-R01).

Acknowledgments: I.J. thanks the Department of Economic Development and Infrastructures of the Basque Government for receiving a PhD grant for young researchers in the scientific-technological and business environment of the Basque agricultural and food sector. This paper is contribution $n^{\circ} 994$ from AZTI, Food Research, Basque Research and Technology Alliance (BRTA).

Conflicts of Interest: C.F. is Scientific Director and cofounder of Lipinutragen srl. born as spin-off company of the Consiglio Nazionale delle Ricerche in Bologna (Italy). 


\section{Abbreviations}

\begin{tabular}{ll} 
AA & Arachidonic Acid \\
ANCOVA & Analysis of Covariance \\
BMI & Body Mass Index \\
DGLA & Dihomo-Gamma-Linolenic Acid \\
DHA & Docosahexaenoic Acid \\
DPA & Docosapentaenoic Acid \\
EDTA & Ethylenediaminetetraacetic Acid \\
EFA & Essential Fatty Acid \\
EPA & Eicosapentaenoic Acid \\
FA & Fatty Acid \\
FFQ & Food Frequency Questionnaire \\
FAME & Fatty Acid Methyl ester \\
KMO & Kaiser-Meyer-Olkin \\
MeOH & Methyl Alcohol \\
MUFA & Monounsaturated Fatty Acids \\
KOH & Potassium Hydroxide \\
LA & Linoleic Acid \\
PUFA & Polyunsaturated Fatty Acid \\
RBC & Red Blood Cell \\
SFA & Saturated Fatty Acid \\
SCD1 & Stearoyl-CoA Desaturase-1 \\
SD & Standard Deviation \\
TFA & Trans Fatty Acid \\
UI & Unsaturation Index \\
PI & Peroxidation Index \\
PCA & Principal Component Analysis \\
& \\
\hline
\end{tabular}

\section{References}

1. Mozaffarian, D. Dietary and Policy Priorities for Cardiovascular Disease, Diabetes, and Obesity: A Comprehensive Review. Circulation 2016, 133, 187-225. [CrossRef]

2. Ng, M.; Fleming, T.; Robinson, M.; Thomson, B.; Graetz, N.; Margono, C.; Mullany, E.C.; Biryukov, S.; Abbafati, C.; Abera, S.F.; et al. Global, regional, and national prevalence of overweight and obesity in children and adults during 1980-2013: A systematic analysis for the Global Burden of Disease Study 2013. Lancet 2014, 384, 766-781. [CrossRef]

3. Murtagh, E. Worldwide trends in body-mass index, underweight, overweight, and obesity from 1975 to 2016 : A pooled analysis of 2416 population-based measurement studies in 128.9 million children, adolescents, and adults. Lancet 2017, 390, 2627-2642.

4. Juonala, M.; Lau, T.; Wake, M.; Grobler, A.; Kerr, J.A.; Magnussen, C.G.; Sabin, M.A.; Burgner, D.P.; Lycett, K. Early clinical markers of overweight/obesity onset and resolution by adolescence. Int. J. Obes. 2020, 44, 82-93. [CrossRef] [PubMed]

5. Beulen, Y.; Martinez-Gonzalez, M.A.; van de Rest, O.; Salas-Salvado, J.; Sorli, J.V.; Gomez-Gracia, E.; Fiol, M.; Estruch, R.; Santos-Lozano, J.M.; Schröder, H.; et al. Quality of Dietary Fat Intake and Body Weight and Obesity in a Mediterranean Population: Secondary Analyses within the PREDIMED Trial. Nutrients 2018, 10, 2011. [CrossRef] [PubMed]

6. Zamanian-Azodi, M.; Vafaee, R.; Azodi, T.; Omidi, R.; Gilanchi, S.; Azizi-Jalilian, F.; Khodarahmi, R. Molecular approaches in obesity studies. Gastroenterol. Hepatol. Bed Bench 2013, 6 (Suppl. 1), S23-S31.

7. Hodson, L.; Skeaff, C.M.; Fielding, B.A. Fatty acid composition of adipose tissue and blood in humans and its use as a biomarker of dietary intake. Prog. Lipid Res. 2008, 47, 348-380. [CrossRef]

8. Viviani Anselmi, C.; Ferreri, C.; Novelli, V.; Roncarati, R.; Bronzini, R.; Marchese, G.; Somalvico, F.; Condorelli, G.; Montenero, A.S.; Puca, A.A. Fatty acid percentage in erythrocyte membranes of atrial flutter/fibrillation patients and controls. J. Interv. Card. Electrophysiol. 2010, 27, 95-99. [CrossRef] 
9. Ghezzo, A.; Visconti, P.; Abruzzo, P.M.; Bolotta, A.; Ferreri, C.; Gobbi, G.; Malisardi, G.; Manfredini, S.; Marini, M.; Nanetti, L. Oxidative Stress and Erythrocyte Membrane Alterations in Children with Autism: Correlation with Clinical Features. PLoS ONE 2013, 8, e66418. [CrossRef]

10. Van Meer, G.; Voelker, D.R.; Feigenson, G.W. Membrane lipids: Where they are and how they behave. Nat. Rev. Mol. Cell Biol. 2008, 9, 112-124. [CrossRef]

11. Ferreri, C.; Masi, A.; Sansone, A.; Giacometti, G.; Larocca, A.V.; Menounou, G.; Scanferlato, R.; Tortorella, S.; Rota, D.; Conti, M.; et al. Fatty Acids in Membranes as Homeostatic, Metabolic and Nutritional Biomarkers: Recent Advancements in Analytics and Diagnostics. Diagnostics 2016, 7, 1. [CrossRef] [PubMed]

12. Ferreri, C.; Chatgilialoglu, C. Membrane Lipidomics for Personalized Health; John Wiley and Sons, Incorporated: Hoboken, NJ, USA, 2015; pp. 87-88.

13. Risé, P.; Eligini, S.; Ghezzi, S.; Colli, S.; Galli, C. Fatty acid composition of plasma, blood cells and whole blood: Relevance for the assessment of the fatty acid status in humans. Prostaglandins Leukot. Essent. Fat. Acids 2007, 76, 363-369. [CrossRef] [PubMed]

14. Sun, Q.; Ma, J.; Campos, H.; Hankinson, S.E.; Hu, F.B. Comparison between plasma and erythrocyte fatty acid content as biomarkers of fatty acid intake in US women. Am. J. Clin. Nutr. 2007, 86, 74-81. [CrossRef] [PubMed]

15. Gunes, O.; Tascilar, E.; Sertoglu, E.; Tas, A.; Serdar, M.A.; Kaya, G.; Kayadibi, H.; Ozcan, O. Associations between erythrocyte membrane fatty acid compositions and insulin resistance in obese adolescents. Chem. Phys. Lipids 2014, 184, 69-75. [CrossRef] [PubMed]

16. Sánchez González, E.; Carrascosa Lezcano, A.; Fernández García, J.M.; Ferrández Longás, A.; López de Lara, D.; López-Siguero, J.P. Estudios españoles de crecimiento: Situación actual, utilidad y recomendaciones de uso. An. Pediatría 2011, 74, 193.e1-193.e16. [CrossRef]

17. Carrascosa, A.; Fernández, J.M.; Fernández, C.; Ferrández, A.; López-Siguero, J.P.; Sánchez, E.; Yeste, D. Estudios españoles de crecimiento 2008. Nuevos patrones antropométricos. Endocrinol. Nutr. 2008, 55, 484-506. [CrossRef]

18. Madrigal, C.; Soto-Mendez, M.J.; Hernandez-Ruiz, A.; Ruiz, E.; Valero, T.; Avila, J.M.; Lara-Villoslada, F.; Leis, R.; Martínez de Victoria, E.; Moreno, J.M.; et al. Dietary and Lifestyle Patterns in the Spanish Pediatric Population (One to < 10 Years Old): Design, Protocol, and Methodology of the EsNuPI Study. Nutrients 2019, 11, 3050.

19. Fernandez-Ballart, J.D.; Pinol, J.L.; Zazpe, I.; Corella, D.; Carrasco, P.; Toledo, E.; Perez-Bauer, M.; Martínez-González, M.Á.; Salas-Salvadó, J.; Martín-Moreno, J.M. Relative validity of a semi-quantitative food-frequency questionnaire in an elderly Mediterranean population of Spain. Br. J. Nutr. 2010, 103, 1808-1816. [CrossRef]

20. Pérez-Farinós, N.; Villar-Villalba, C.; López Sobaler, A.M.; Dal Re Saavedra, M.; Aparicio, A.; Santos Sanz, S.; de Dios, T.R.; Castrodeza-Sanz, J.J.; Anta, R.M. The relationship between hours of sleep, screen time and frequency of food and drink consumption in Spain in the 2011 and 2013 ALADINO: A cross-sectional study. BMC Public Health 2017, 17, 1-12. [CrossRef]

21. Stefan, L.; Prosoli, R.; Juranko, D.; Cule, M.; Milinovic, I.; Novak, D.; Sporiš, G. The Reliability of the Mediterranean Diet Quality Index (KIDMED) Questionnaire. Nutrients 2017, 9, 419. [CrossRef]

22. Serra-Majem, L.; Ribas, L.; Ngo, J.; Ortega, R.M.; Garcia, A.; Perez-Rodrigo, C.; Aranceta, J. Food, youth and the Mediterranean diet in Spain. Development of KIDMED, Mediterranean Diet Quality Index in children and adolescents. Public Health Nutr. 2004, 7, 931-935. [CrossRef] [PubMed]

23. Sansone, A.; Tolika, E.; Louka, M.; Sunda, V.; Deplano, S.; Melchiorre, M.; Anagnostopoulos, D.; Chatgilialoglu, C.; Formisano, C.; Di Micco, R.; et al. Hexadecenoic Fatty Acid Isomers in Human Blood Lipids and Their Relevance for the Interpretation of Lipidomic Profiles. PLoS ONE 2016, 11, e0152378. [CrossRef] [PubMed]

24. Giacometti, G.; Ferreri, C.; Sansone, A.; Chatgilialoglu, C.; Marzetti, C.; Spyratou, E.; Georgakilas, A.G.; Marini, M.; Abruzzo, P.M.; Bolotta, A.; et al. High predictive values of RBC membrane-based diagnostics by biophotonics in an integrated approach for Autism Spectrum Disorders. Sci. Rep. 2017, 7, 9854. [CrossRef] [PubMed]

25. Amezaga, J.; Arranz, S.; Urruticoechea, A.; Ugartemendia, G.; Larraioz, A.; Louka, M.; Uriarte, M.; Ferreri, C.; Tueros, I. Altered Red Blood Cell Membrane Fatty Acid Profile in Cancer Patients. Nutrients 2018, 10, 1853. [CrossRef] 
26. Van Der Vegt, S.G.L.; Ruben, A.M.T.; Werre, J.M.; Palsma, D.M.H.; Verhoef, C.W.; de Gier, J.; Staal, G.E. Counterflow centrifugation of red cell populations: A cell age related separation technique. Br. J. Haematol. 1985, 61, 393-403. [CrossRef]

27. Breil, C.; Abert Vian, M.; Zemb, T.; Kunz, W.; Chemat, F. "Bligh and Dyer" and Folch Methods for Solid-Liquid-Liquid Extraction of Lipids from Microorganisms. Comprehension of Solvatation Mechanisms and towards Substitution with Alternative Solvents. Int. J. Mol. Sci. 2017, 18, 708. [CrossRef]

28. Ferreri, C.; Faraone Mennella, M.R.; Formisano, C.; Landi, L.; Chatgilialoglu, C. Arachidonate geometrical isomers generated by thiyl radicals: The relationship with trans lipids detected in biological samples. Free Radic. Biol. Med. 2002, 33, 1516-1526. [CrossRef]

29. Cohen, J. Statistical Power Analysis. Curr. Dir. Psychol. Sci. 1992, 1, 98-101. [CrossRef]

30. Choi, H.-N.; Yim, J.-E. Effects of Erythrocyte Membrane Polyunsaturated Fatty Acids in Overweight, Obese, and Morbidly Obese Korean Women. J. Cancer Prev. 2017, 22, 182-188. [CrossRef]

31. Genio, G. Morbid Obesity is Associated to Altered Fatty Acid Profile of Erythrocyte Membranes. J. Diabetes Metab. 2015, 6, 1-4.

32. Bonafini, S.; Tagetti, A.; Gaudino, R.; Cavarzere, P.; Montagnana, M.; Danese, E.; Benati, M.; Ramaroli, D.A.; Raimondi, S.; Giontella, A.; et al. Individual fatty acids in erythrocyte membranes are associated with several features of the metabolic syndrome in obese children. Eur. J. Nutr. 2019, 58, 731-742. [CrossRef] [PubMed]

33. Fekete, K.; Györei, E.; Lohner, S.; Verduci, E.; Agostoni, C.; Decsi, T. Long-chain polyunsaturated fatty acid status in obesity: A systematic review and meta-analysis. Obes. Rev. Off. J. Int. Assoc. Study Obes. 2015, 16, 488-497. [CrossRef] [PubMed]

34. Araya, J.; Rodrigo, R.; Videla, L.A.; Thielemann, L.; Orellana, M.; Pettinelli, P. Increase in long-chain polyunsaturated fatty acid $n-6 / n-3$ ratio in relation to hepatic steatosis in patients with non-alcoholic fatty liver disease. Clin. Sci. 2004, 106, 635-643. [CrossRef] [PubMed]

35. Savva, S.C.; Chadjigeorgiou, C.; Hatzis, C.; Kyriakakis, M.; Tsimbinos, G.; Tornaritis, M.; Kafatos, A. Association of adipose tissue arachidonic acid content with BMI and overweight status in children from Cyprus and Crete. Br. J. Nutr. 2004, 91, 643-649. [CrossRef] [PubMed]

36. Pietiläinen, K.H.; Róg, T.; Seppänen-Laakso, T.; Virtue, S.; Gopalacharyulu, P.; Tang, J.; Rodriguez-Cuenca, S.; Maciejewski, A.; Naukkarinen, J.; Ruskeepää, A.L.; et al. Association of lipidome remodeling in the adipocyte membrane with acquired obesity in humans. PLoS Biol. 2011, 9, 7. [CrossRef]

37. Stark, K.D.; Van Elswyk, M.E.; Higgins, M.R.; Weatherford, C.A.; Salem, N. Global survey of the omega-3 fatty acids, docosahexaenoic acid and eicosapentaenoic acid in the blood stream of healthy adults. Prog. Lipid Res. 2016, 63, 132-152. [CrossRef]

38. Sonnweber, T.; Pizzini, A.; Nairz, M.; Weiss, G.; Tancevski, I. Arachidonic Acid Metabolites in Cardiovascular and Metabolic Diseases. Int. J. Mol. Sci. 2018, 19, 3285. [CrossRef]

39. Burns, J.L.; Nakamura, M.T.; Ma, D.W.L. Differentiating the biological effects of linoleic acid from arachidonic acid in health and disease. Prostaglandins Leukot. Essent. Fat. Acids 2018, 135, 1-4. [CrossRef]

40. Ailhaud, G.; Guesnet, P.; Cunnane, S.C. An emerging risk factor for obesity: Does disequilibrium of polyunsaturated fatty acid metabolism contribute to excessive adipose tissue development? Br. J. Nutr. 2008, 100, 461-470. [CrossRef]

41. Muhlhausler, B.S.; Ailhaud, G.P. Omega-6 polyunsaturated fatty acids and the early origins of obesity. Curr. Opin. Endocrinol. Diabetes Obes. 2013, 20, 56-61. [CrossRef]

42. Simopoulos, A.P. An Increase in the Omega-6/Omega-3 Fatty Acid Ratio Increases the Risk for Obesity. Nutrients 2016, 8, 128. [CrossRef] [PubMed]

43. Cao, H.; Gerhold, K.; Mayers, J.R.; Wiest, M.M.; Watkins, S.M.; Hotamisligil, G.S. Identification of a lipokine, a lipid hormone linking adipose tissue to systemic metabolism. Cell 2008, 134, 933-944. [CrossRef] [PubMed]

44. Warensjo, E.; Ohrvall, M.; Vessby, B. Fatty acid composition and estimated desaturase activities are associated with obesity and lifestyle variables in men and women. Nutr. Metab. Cardiovasc. Dis. 2006, 16, 128-136. [CrossRef] [PubMed]

45. Puri, P.; Wiest, M.M.; Cheung, O.; Mirshahi, F.; Sargeant, C.; Min, H.K.; Contos, M.J.; Sterling, R.K.; Fuchs, M.; Zhou, H.; et al. The plasma lipidomic signature of nonalcoholic steatohepatitis. Hepatology 2009, 50, 1827-1838. [CrossRef] [PubMed] 
46. Sjogren, P.; Sierra-Johnson, J.; Gertow, K.; Rosell, M.; Vessby, B.; de Faire, U.; Hamsten, A.; Hellenius, M.L.; Fisher, R.M. Fatty acid desaturases in human adipose tissue: Relationships between gene expression, desaturation indexes and insulin resistance. Diabetologia 2008, 51, 328-335. [CrossRef]

47. Warensjo, E.; Riserus, U.; Vessby, B. Fatty acid composition of serum lipids predicts the development of the metabolic syndrome in men. Diabetologia 2005, 48, 1999-2005. [CrossRef]

48. Notarnicola, M.; Caruso, M.G.; Tutino, V.; Bonfiglio, C.; Cozzolongo, R.; Giannuzzi, V.; De Nunzio, V.; De Leonardis, G.; Abbrescia, D.I.; Franco, I.; et al. Significant decrease of saturation index in erythrocytes membrane from subjects with non-alcoholic fatty liver disease (NAFLD). Lipids Health Dis. 2017, 16, 1-7. [CrossRef]

49. Weijers, R. Membrane flexibility, free fatty acids, and the onset of vascular and neurological lesions in type 2 diabetes. J. Diabetes Metab. Disord. 2016, 15, 13. [CrossRef]

50. De Toro-Martín, J.; Arsenault, B.J.; Després, J.P.; Vohl, M.C. Precision Nutrition: A Review of Personalized Nutritional Approaches for the Prevention and Management of Metabolic Syndrome. Nutrients 2017, 9, 913. [CrossRef]

51. Abbaspour, N.; Roberts, T.; Hooshmand, S.; Kern, M.; Hong, M.Y. Mixed Nut Consumption May Improve Cardiovascular Disease Risk Factors in Overweight and Obese Adults. Nutrients 2019, 11, 1488. [CrossRef]

52. Rock, C.L.; Flatt, S.W.; Barkai, H.S.; Pakiz, B.; Heath, D.D. Walnut consumption in a weight reduction intervention: Effects on body weight, biological measures, blood pressure and satiety. Nutr. J. 2017, 16, 76. [CrossRef] [PubMed]

53. Razquin, C.; Sanchez-Tainta, A.; Salas-Salvado, J.; Buil-Cosiales, P.; Corella, D.; Fito, M.; Ros, E.; Estruch, R.; Arós, F.; Gómez-Gracia, E.; et al. Dietary energy density and body weight changes after 3 years in the PREDIMED study. Int. J. Food Sci. Nutr. 2017, 68, 865-872. [CrossRef] [PubMed]

54. Kim, Y.; Keogh, J.B.; Clifton, P.M. Benefits of Nut Consumption on Insulin Resistance and Cardiovascular Risk Factors: Multiple Potential Mechanisms of Actions. Nutrients 2017, 9, 1271. [CrossRef] [PubMed]

55. Ros, E. Health benefits of nut consumption. Nutrients 2010, 2, 652-682. [CrossRef] [PubMed]

56. Iaccarino Idelson, P.; Scalfi, L.; Valerio, G. Adherence to the Mediterranean Diet in children and adolescents: A systematic review. Nutr. Metab. Cardiovasc. Dis. 2017, 27, 283-299. [CrossRef] [PubMed]

57. Jennings, A.; Welch, A.; van Sluijs, E.M.; Griffin, S.J.; Cassidy, A. Diet quality is independently associated with weight status in children aged 9-10 years. J. Nutr. 2011, 141, 453-459. [CrossRef]

58. Lydakis, C.; Stefanaki, E.; Stefanaki, S.; Thalassinos, E.; Kavousanaki, M.; Lydaki, D. Correlation of blood pressure, obesity, and adherence to the Mediterranean diet with indices of arterial stiffness in children. Eur. J. Pediatr. 2012, 171, 1373-1382. [CrossRef]

59. Saravia, L.; Miguel-Berges, M.L.; Iglesia, I.; Nascimento-Ferreira, M.V.; Perdomo, G.; Bove, I.; Slater, B.; Moreno, L.A. Relative validity of FFQ to assess food items, energy, macronutrient and micronutrient intake in children and adolescents: A systematic review with meta-analysis. Br. J. Nutr. 2020, 18, 1-27. [CrossRef]

Publisher's Note: MDPI stays neutral with regard to jurisdictional claims in published maps and institutional affiliations.

(C) 2020 by the authors. Licensee MDPI, Basel, Switzerland. This article is an open access article distributed under the terms and conditions of the Creative Commons Attribution (CC BY) license (http://creativecommons.org/licenses/by/4.0/). 